PROCEEDINGS OF THE

AMERICAN MATHEMATICAL SOCIETY

Volume 128, Number 4, Pages 1097-1103

S 0002-9939(99)05104-7

Article electronically published on August 5, 1999

\title{
EXTREME POINTS OF THE UNIT BALL OF THE FOURIER-STIELTJES ALGEBRA
}

\author{
PETER F. MAH AND TIANXUAN MIAO
}

(Communicated by Dale Alspach)

Dedicated to Professor Edmond E. Granirer, with our admiration and respect, on the occasion of his retirement

\begin{abstract}
Let $G$ be a locally compact group. Among other things, we proved in this paper that for an IN-group $G$, the extreme points of the unit ball of the Fourier-Stieltjes algebra $B(G)$ are not in the Fourier algebra $A(G)$ if and only if $G$ is non-compact, or equivalently, there is no irreducible representation of $G$ which is quasi-equivalent to a subrepresentation of the left regular representation of $G$ if and only if $G$ is non-compact. This result is a non-commutative version of the following well known result: For any locally compact group $\widehat{G}$, the extreme points of the unit ball of the measure algebra $M(\widehat{G})$ are not in the group algebra $L^{1}(\widehat{G})$ if and only if $\widehat{G}$ is non-discrete. On the other hand, we also showed that if $B(G)$ has the RNP, then there are extreme points of the unit ball of $B(G)$ that are in $A(G)$. Since it is well known there are noncompact locally compact group $G$ for which $B(G)$ has the RNP, there exist non-compact locally compact groups $G$ where extreme points of the unit ball of $B(G)$ can be in $A(G)$. This shows that the condition $G$ be an IN-group cannot be entirely removed.
\end{abstract}

\section{INTRODUCTION}

Let $\widehat{G}$ be a locally compact group. Let $L^{1}(\widehat{G})$ and $M(\widehat{G})$ be the group algebra and the measure algebra of $\widehat{G}$, respectively. It is well known that the extreme points of the unit ball of $M(\widehat{G})$ are not in $L^{1}(\widehat{G})$ if and only if $\widehat{G}$ is non-discrete. Now, if $\widehat{G}$ is the dual group of a locally compact Abelian group $G$, then it is well known that $A(G)$, the Fourier algebra of $G$, and $B(G)$, the Fourier-Stieltjes algebra of $G$, are isometrically isomorphic to $L^{1}(\widehat{G})$ and $M(\widehat{G})$, respectively, and that $G$ is non-compact if and only if $\widehat{G}$ is non-discrete. Thus the above result can be recast as follows: For an Abelian group $G$, the extreme points of the unit ball of $B(G)$ do not lie in $A(G)$ if and only if $G$ is non-compact. Our main purpose in this paper is to investigate the non-commutative version of this result. Among other results, we showed that if $G$ is an IN-group, then the extreme points of the unit ball of $B(G)$ do not lie in $A(G)$ if and only if $G$ is non-compact. Since the class of IN-groups

Received by the editors November 14, 1997 and, in revised form, June 1, 1998.

1991 Mathematics Subject Classification. Primary 43A30, 43A35, 43A65, 22D99.

Key words and phrases. Locally compact groups, extreme points, weak*-strongly exposed points, Fourier algebra, Fourier-Stieltjes algebra.

This research is supported by an NSERC grant. 
contains all Abelian groups, our result constitutes an extension of the above result to non-commutative groups. Equivalently, we can state our result in terms of group representation: For an IN-group $G$, there is no irreducible representation of $G$ which is (quasi)-equivalent to a subrepresentation of the left regular representation of $G$ if and only if $G$ is non-compact.

Our results can be summarized as follows (see section 2 for definitions). For an IN-group $G$, the following conditions are equivalent: (1) $G$ is compact; (2) $\operatorname{Ext}\left(B(G)^{1}\right) \cap A(G) \neq \emptyset$; (3) For the left regular representation $\rho$ of $G, A(G)=$ $B_{\rho}(G)$; (4) $A(G)$ is weak*-closed in $B(G)$; (5) For some continuous unitary representation $\pi$ of $G, B_{\pi}(G) \subset A(G)$; $(6) B(G)$ has the RNP; (7) For the left regular representation $\rho$ of $G, B_{\rho}(G)$ has the RNP. On the other hand, we proved that for any locally compact group $G$, if $B(G)$ has the RNP, then there are elements in $A(G)$ which are extreme points of the unit ball of $B(G)$. Since it is well known that there are non-compact groups $G$ (for example, Fell's group) for which $B(G)$ has the RNP, we see that our main result cannot be extended to a general locally compact group. In proving our main result, we also proved a result which is interesting by itself, namely that for any locally compact group $G$, the set of weak*-strongly exposed points of the set of norm decreasing functionals in $P_{\rho}(G)$ are in $A(G)$.

\section{Preliminaries}

If $X$ is a Banach space, we denote the dual Banach space of $X$ by $X^{*}$ and the space of continuous linear operators from $X$ into $X$ by $\mathcal{B}(X)$. For $x \in X$ and $f \in X^{*}$, the value $f(x)$ is sometimes denoted by $\langle f, x\rangle$ or $\langle x, f\rangle$. The unit ball of $X$ will be denoted by $X^{1}$. More generally, if $A \subset X$, the set of those elements of $A$ with norm at most equal to one is denoted by $A^{1}$, and the set of extreme points of $A$ is denoted by $\operatorname{Ext}(A)$. For a convex subset $E$ in $X^{*}$, an element $f_{0} \in E$ is called a weak*-strongly exposed point of $E$ if there exists an element $x_{0} \in X$ such that $f\left(x_{0}\right)<f_{0}\left(x_{0}\right)$ for all $f \in E \backslash\left\{f_{0}\right\}$ and whenever $f_{n} \in E$ and $f_{n}\left(x_{0}\right) \rightarrow f_{0}\left(x_{0}\right)$, then $\left\|f_{n}-f_{0}\right\| \rightarrow 0$. For the definition of and results on the Radon-Nikodym property (denoted in short by RNP), we refer the readers to [4].

Let $G$ be a locally compact group with a fixed left Haar measure. Let $A(G)$ and $B(G)$ be the Fourier and Fourier-Stieltjes algebras of $G$, respectively, as defined in Eymard [6]. As usual, $L^{1}(G)$ denotes the group algebra, $M(G)$ denotes the measure algebra and $C^{*}(G)$ denotes the group $C^{*}$-algebra of $G$. It is well known that $B(G)$ is the dual Banach space of $C^{*}(G)$, and that $A(G)$ is a two-sided ideal of $B(G)$. See [6] for more basic properties of these spaces.

We refer the reader to Dixmier [5] for definitions and basic properties of (unitary) representations of $G$. Given a representation $\pi$ of $G$, its representation space is denoted by $H_{\pi}$. For $\xi, \eta \in H_{\pi}$, the function $a_{\xi, \eta}$, denoted also by the symbol $\xi *_{\pi} \eta$, defined on $G$ by $a_{\xi, \eta}(x)=\langle\pi(x) \xi, \eta\rangle$ is called a coefficient function of $\pi$. Here, $\langle\cdot, \cdot\rangle$ denotes the inner product in $H_{\pi}$. The closed linear span of $\left\{a_{\xi, \eta}: \xi, \eta \in H_{\pi}\right\}$ in $B(G)$ is denoted by $A_{\pi}(G)$, and the weak*-closure of $A_{\pi}(G)$ in $B(G)$ is denoted by $B_{\pi}(G)$. The dual of $A_{\pi}(G)$ is denoted by $V N_{\pi}(G)$. It is well known that $V N_{\pi}(G)$ is the von Neumann algebra generated by $\{\pi(x): x \in G\}$ in $\mathcal{B}\left(H_{\pi}\right)$.

Each representation $\pi$ of $G$ can be extended to a representation on $M(G)$ by means of the formula $\pi(\mu)=\int_{G} \pi(x) d \mu(x)$ for all $\mu \in M(G)$. Note that if $\delta_{x}$ denotes the point measure at $x$, we have $\pi\left(\delta_{x}\right)=\pi(x)$. If we restrict the representation to $L^{1}(G)$, then we get a non-degenerate representation of $L^{1}(G)$. This 
representation can be lifted to a representation on $C^{*}(G)$, which is usually denoted by $\pi$ again. If $N_{\pi}$ denotes the kernel of this representation, then we set $C_{\pi}^{*}(G):=C^{*}(G) / N_{\pi}$. Then $C_{\pi}^{*}(G)$ is a $C^{*}$-algebra and its dual Banach space is $B_{\pi}(G)$.

Throughout this paper, we reserve the symbol $\rho$ to denote the left regular representation of $G$. Note that $A_{\rho}(G)=A(G)$ and $V N_{\rho}(G)=V N(G)$. The space $C_{\rho}^{*}(G)$ is called the reduced group $C^{*}$-algebra of $G$, and $B_{\rho}(G)=C_{\rho}^{*}(G)^{*}$.

We denote the set of continuous positive definite functions on $G$ by $P(G)$. Then, as is well known, $P(G)$ can be identified with the set of positive linear functionals on $C^{*}(G)$. The set of continuous positive linear functionals on $C_{\rho}^{*}(G)$ is denoted by $P_{\rho}(G)$, and we have $P_{\rho}(G)=P(G) \cap B_{\rho}(G)$. We refer the reader to Arsac [1] and Eymard [6] for more properties.

A locally compact group $G$ is called an IN-group if it has a compact neighbourhood of the identity $e \in G$ which is invariant under inner automorphisms. It is called a SIN-group if it has a base for the neighbourhood system of $e$ consisting of compact sets invariant under inner automorphisms. If [IN] denotes the class of IN-group, etc., then we have

$$
[\mathrm{X}] \subset[\mathrm{SIN}] \subset[\mathrm{IN}] \subset[\text { unimodular }]
$$

where $\mathrm{X}$ is discrete, Abelian, or compact. We refer the reader to the survey paper by Palmer [12] and the references therein for more on these groups.

\section{On EXTREME POINTS}

In preparation for the proof of the following theorem, we need some tools which will allow us to reduce arguments on a group to arguments on a quotient group.

Suppose $G$ is a locally compact group and $K$ is a compact normal subgroup of $G$. Let $\pi$ be a unitary representation of $G$ with representation space $H_{\pi}$. Then $\pi$ induces a unitary representation $\dot{\pi}$ on $G / K$ as follows. First, we define a projection $P$ on $H_{\pi}$ by

$$
\langle P \xi, \eta\rangle=\int_{K}\langle\pi(x) \xi, \eta\rangle d x, \quad \xi, \eta \in H_{\pi} .
$$

Then $\dot{\pi}$, defined by

$$
\langle\dot{\pi}(\dot{x}) \xi, \eta\rangle=\int_{K}\langle\pi(t x) \xi, \eta\rangle d t, \quad \dot{x} \in G / K, \xi, \eta \in P H_{\pi},
$$

is a continuous unitary representation of $G / K$ with representation space $P H_{\pi}$. Moreover, we have $\dot{\pi}(\dot{x}) \xi=\pi(x) \xi$ for all $\xi \in P H_{\pi}$. See [10] Proposition 3.4] for details.

It is well known that $A(G / K)$ is isometrically isomorphic to the subalgebra $A_{K}(G)$ of $A(G)$, consisting of functions that are constant on the cosets of $K$. For each $a \in A(G)$, we define the function $\dot{a}$ on $G / K$ by

$$
\dot{a}(\dot{x})=\int_{K} a(x k) d k .
$$

By Proposition 6 in [7], the map $a \rightarrow \dot{a}$ is a Banach space retraction from $A(G)$ to $A(G / K)$. Moreover, as the proof of Corollary 3.5 in [10] shows, we have

$$
\dot{a}(\dot{x})=\langle\dot{\pi}(\dot{x}) \xi, \eta\rangle, \quad \xi, \eta \in P H_{\pi} .
$$


We will need to use the fact that $A(G)$, being the predual of the von Neumann algebra $V N(G)$, has a module structure, which can be described generally as follows. If $M$ is a von Neumann algebra and $M_{*}$ is its predual, then we put on $M_{*}$ a left $M$-module structure if, for each $T \in M$ and $u \in M_{*}$, we define the element $T \cdot u$ in $M_{*}$ by the formula $\langle T \cdot u, S\rangle=\langle u, S T\rangle, \quad S \in M$. A right $M$-module structure and a two-sided $M$-module structure can be similarly defined on $M_{*}$. In the proof below, if $\pi$ is a representation of $G, x, y \in G$ and $a \in A(G)$, we will write $\delta_{x} \cdot a \cdot \delta_{y}$ for $\pi\left(\delta_{x}\right) \cdot a \cdot \pi\left(\delta_{y}\right)$.

3.1. Theorem. Let $G$ be an IN-group, $\left(\pi, H_{\pi}\right)$ be any irreducible representation of $G$ and $\xi, \eta$ be unit vectors in $H_{\pi}$. Then the element $a \in B(G)$ defined by $a(x)=$ $\langle\pi(x) \xi, \eta\rangle$ is not in $A(G)$ if $G$ is not compact.

Proof. Suppose $G$ is not compact. We assume first that $G$ is a SIN-group. Suppose $a \in A(G)$ and $a \neq 0$. Then there exists an $x_{0}$ such that $\epsilon_{0}:=a\left(x_{0}\right) \neq 0$. Since $\pi$ is irreducible, the commutant $\pi\left(C^{*}(G)\right)^{\prime}=\pi\left(L^{1}(G)\right)^{\prime}$ in $\mathcal{B}\left(H_{\pi}\right)$ is equal to $\mathcal{C} 1$, the set of constants. It follows from von Neumann's double commutant theorem that $V N_{\pi}(G)=\pi\left(L^{1}(G)\right)^{\prime \prime}=\mathcal{B}\left(H_{\pi}\right)$ and the subalgebra $\mathcal{K}\left(H_{\pi}\right)$ of compact operators on $H_{\pi}$ is dense in $\mathcal{B}\left(H_{\pi}\right)$ in the $\sigma\left(V N_{\pi}(G), A_{\pi}(G)\right)$-topology [11, Theorem 4.1.5, p.116].

Since $G$ is not compact, by Lemma 3.1 in 9 there is a sequence $x_{n} \in G$ such that " $x_{n} \rightarrow \infty$ " in the sense that, for any compact set $K$ of $G$, there is an $N$ such that $x_{n} \notin K$ for all $n>N$. Furthermore, for each $a \in A(G)$, we have $\delta_{x_{n}} \cdot a \rightarrow 0$ weakly in $A(G)$. Since $G$ is a SIN-group, $V N(G)$ is a finite von Neumann algebra [13, Proposition 4.1]. It follows that $\left\{\delta_{x_{n}} \cdot a \cdot \delta_{x_{0} x_{n}-1}\right\}$ is relatively weakly compact in $A(G)$ [8, Corollary 7.5]. We assume, without loss of generality, that $\delta_{x_{n}} \cdot a \cdot \delta_{x_{0} x_{n}-1} \rightarrow a_{0}$ weakly in $A(G)$. Since $A_{\pi}(G)$ is weakly closed in $A(G)$, $a_{0} \in A_{\pi}(G)$ and $\delta_{x_{n}} \cdot a \cdot \delta_{x_{0} x_{n}-1} \rightarrow a_{0}$ weakly in $A_{\pi}(G)$. Also, we have

$$
\left\langle a_{0}, \delta_{e}\right\rangle=\lim \left\langle\delta_{x_{n}} \cdot a \cdot \delta_{x_{0} x_{n}-1}, \delta_{e}\right\rangle=\lim \left\langle a, \delta_{x_{0} x_{n}-1} \cdot \delta_{e} \cdot \delta_{x_{n}}\right\rangle=\left\langle a, \delta_{x_{0}}\right\rangle=\epsilon_{0} .
$$

Hence $a_{0} \neq 0$, and by the density of $\mathcal{K}\left(H_{\pi}\right)$ in $\mathcal{B}\left(H_{\pi}\right)$ in the $\sigma\left(V N_{\pi}(G), A_{\pi}(G)\right)$ topology there exists a $k \in \mathcal{K}\left(H_{\pi}\right)$ such that $\left\langle k, a_{0}\right\rangle=1$.

For each $n$, we have $k \cdot \delta_{x_{n}} \cdot a=\left(k\left(\delta_{x_{n}} \xi\right)\right) *_{\pi} \eta[1$ (2.7)]. Since $k$ is a compact operator on $H_{\pi}$ and $\left\{\delta_{x_{n}} \xi\right\}$ is bounded, we may assume, without loss of generality, the existence of some $\xi_{0} \in H_{\pi}$ for which $k\left(\delta_{x_{n}} \xi\right) \rightarrow \xi_{0}$ in the norm topology of $H_{\pi}$. Hence $k \cdot \delta_{x_{n}} \cdot a \rightarrow \xi_{0} *_{\pi} \eta$ in the norm topology of $A_{\pi}(G)$. Since the sequence $\left(x_{n}\right)$ that we have chosen also satisfies the property that $\delta_{x_{n}} \cdot a \rightarrow 0$ weakly in $A(G)$, and so in $A_{\pi}(G)$, it follows that, for each $x \in G$,

$$
\xi_{0} *_{\pi} \eta(x)=\left\langle\xi_{0} *_{\pi} \eta, \delta_{x}\right\rangle=\lim \left\langle k \cdot \delta_{x_{n}} \cdot a, \delta_{x}\right\rangle=\lim \left\langle\delta_{x_{n}} \cdot a, \delta_{x} \cdot k\right\rangle=0 .
$$

Consequently we have $\xi_{0} *_{\pi} \eta=0$, and so $\left\|k \cdot \delta_{x_{n}} \cdot a\right\| \rightarrow 0$.

On the other hand, for each $n$,

$$
\left\|k \cdot \delta_{x_{n}} \cdot a\right\| \geq\left\langle k \cdot \delta_{x_{n}} \cdot a, \delta_{x_{0} x_{n}-1}\right\rangle=\left\langle\delta_{x_{n}} \cdot a \cdot \delta_{x_{0} x_{n}-1}, k\right\rangle \rightarrow\left\langle a_{0}, k\right\rangle=1,
$$

which yields a contradiction. Thus, $a$ is not in $A(G)$.

Now assume $G$ is an IN-group. Then there exists a compact normal subgroup $K$ of $G$ such that $G / K$ is a SIN-group [12, diagram 1, p.698], and since $G$ is not compact, $G / K$ is not compact. Suppose $a \in A(G)$. Then, as we observed in the paragraphs before this theorem, the function $a$ induces a function $\dot{a} \in A(G / K)$. Also the representation $\pi$ on $G$ induces a representation $\dot{\pi}$ on 
$G / K$. Moreover, $\dot{\pi}$ is irreducible since $\pi$ is. As we observed earlier, we have $\dot{a}(\dot{x})=\langle\dot{\pi}(\dot{x}) P \xi, P \eta\rangle$ for all $\dot{x} \in G / K, \xi, \eta \in H_{\pi}$. Therefore it follows from the first part of the proof that $G / K$ is a compact group because $G / K$ is a SIN-group. This is a contradiction. Thus $a \notin A(G)$.

Remark. Suppose $\pi$ is an irreducible representation of $G$ with representation space $H_{\pi}$. Then any nonzero $\xi \in H_{\pi}$ is a cyclic vector. Thus $a(x)=\langle\pi(x) \xi, \eta\rangle$ for any $\xi, \eta$ on the unit sphere of $H_{\pi}$ defines an element of $A(G)$ if and only if $A_{\pi}(G) \subset A(G)$. Therefore, by Theorem 3.1, there is no irreducible representation of $G$ which is quasi-equivalent to a subrepresentation of the left regular representation $\rho$ of any non-compact IN-group $G$. See [1, (3.14)].

Arsac showed in [1] that, for any locally compact group $G, B(G)$ can be decomposed into a direct sum $B(G)=A(G) \bigoplus B^{s}(G)$ for some subspace $B^{s}(G)$ of $B(G)$. Since $B(G)=A_{\omega}(G)$, where $\omega$ is the universal representation of $G$ [3 p.393], it follows from Proposition 1 of [3] that every extreme point $a$ of the unit ball of $B(G)$ is of the form $a(x)=\langle\pi(x) \xi, \eta\rangle$ for some irreducible reprsentation $\left(\pi, H_{\pi}\right)$ of $G$, and some $\xi, \eta \in H_{\pi}$. Thus it follows from Theorem 3.1 that, for a non-compact IN-group $G$, any extreme points $b$ of the unit ball of $B(G)$ must be in $B^{s}(G)$. By the characterization of the elements of $B^{s}(G)$ in Miao [ 10 ], we have the following result.

3.2. Corollary. Let $G$ be a non-compact IN-group. If $b \in \operatorname{Ext}\left(B(G)^{1}\right)$, then $b \in$ $B^{s}(G)$. Consequently, $b$ has the following property: for any $\epsilon>0$ and any compact subset $K \subset G$, there is an $f \in L^{1}(G)$ with $\|f\|_{C^{*}(G)} \leq 1$ and $\operatorname{supp}(f) \subset G \backslash K$ such that $|\langle f, b\rangle|>\|b\|-\epsilon$.

3.3. Theorem. Let $G$ be a locally compact group. Then all the weak*-strongly exposed points of $P_{\rho}(G)^{1}$ are in $A(G)$.

Proof. Let $b_{0}$ be a weak ${ }^{*}$-strongly exposed points of $P_{\rho}(G)^{1}$. Then $b_{0}=\lim f_{\alpha} * \tilde{f}_{\alpha}$ uniformly on compact subsets of $G$ [6] (1.25)], where $f_{\alpha} \in L^{2}(G)$ for all $\alpha$. Hence

$$
1=b_{0}(e)=\lim \left(f_{\alpha} * \tilde{f}_{\alpha}\right)(e)=\lim \left\|f_{\alpha} * \tilde{f}_{\alpha}\right\|_{A(G)} .
$$

We assume, without loss of generality, that $\left\|f_{\alpha} * \tilde{f}_{\alpha}\right\|_{A(G)}=1$ for all $\alpha$. Note that $f_{\alpha} * \tilde{f}_{\alpha} \in A(G)$ and $b_{0}$ is the limit of the net $\left\{f_{\alpha} * \tilde{f}_{\alpha}\right\}$ in the weak*-topology. It follows from the fact that $b_{0}$ is a weak* ${ }^{*}$-strongly exposed point of $P_{\rho}(G)^{1}$ that $\left\|f_{\alpha} * \tilde{f}_{\alpha}-b_{0}\right\|_{A(G)} \rightarrow 0$. Therefore $b_{0} \in A(G)$.

3.4. Theorem. Let $G$ be an IN-group. Then the following statements are equivalent.

(a) $G$ is compact.

(b) $\operatorname{Ext}\left(B(G)^{1}\right) \cap A(G) \neq \emptyset$.

(c) $A(G)=B_{\rho}(G)$.

(d) $A(G)$ is weak ${ }^{*}$-closed in $B(G)$.

(e) For some continuous unitary representation $\pi$ of $G, B_{\pi}(G) \subset A(G)$.

(f) $B(G)$ has the RNP.

(g) $B_{\rho}(G)$ has the $R N P$.

Proof. (a) $\Rightarrow$ (b) If $G$ is compact, then $A(G)=B(G)$. Hence $\operatorname{Ext}\left(B(G)^{1}\right) \subseteq A(G)$, and so $\operatorname{Ext}\left(B(G)^{1}\right) \cap A(G) \neq \varnothing$. 
(b) $\Rightarrow$ (a) Let $a \in \operatorname{Ext}\left(B(G)^{1}\right) \cap A(G)$ and suppose $G$ is noncompact. Let $\omega$ be the universal representation of $G$ (or $C^{*}(G)$ ). Then $B(G)^{1}=A_{\omega}(G)^{1}[3$ p. 393], and so by Proposition 1 of Bélanger and Forrest [3], there exist an irreducible representation $\pi$ of $G$ on a Hilbert space $H_{\pi}$ and some $\xi$ and $\eta$ on the unit sphere of $H_{\pi}$ such that $a(x)=\langle\pi(x) \xi, \eta\rangle$ for all $x \in G$. By Theorem 3.1, $a \notin A(G)$. This is impossible since $a \in A(G)$. Thus $G$ must be compact.

(c) $\Leftrightarrow(\mathrm{d})$ and $(\mathrm{a}) \Rightarrow(\mathrm{c})$ are well known.

(c) $\Rightarrow$ (e) is trivial.

(e) $\Rightarrow$ (a) If $\pi_{\omega}$ is the universal representation of $C_{\pi}^{*}(G)$, then $A_{\pi_{\omega}}(G)=B_{\pi}(G)$ [3, p. 393]. Since $B_{\pi}(G)=C_{\pi}^{*}(G)^{*}$, there is an extreme point $a$ on the unit ball of $B_{\pi}(G)$. By Proposition 1 of Bélanger and Forrest [3], there is an irreducible subrepresentation $\left(\tau, H_{\tau}\right)$ of $\pi_{\omega}$ and some $\xi$ and $\eta$ on the unit sphere of $H_{\tau}$ such that $a(x)=\langle\tau(x) \xi, \eta\rangle$ for all $x \in G$. It follows from Theorem 3.1 that $G$ is compact.

(a) $\Rightarrow$ (f) is well known and (f) $\Rightarrow(\mathrm{g})$ is trivial since $B_{\rho}(G) \subset B(G)$.

$(\mathrm{g}) \Rightarrow(\mathrm{b})$ Suppose $B_{\rho}(G)$ has the RNP. Then by [4, p.213] $P_{\rho}(G)^{1}$ has a weak*strongly exposed point, say $a$. By Theorem 3.3, $a \in A(G)$. We will show that $a$ is also an extreme point of the unit ball of $B(G)$.

Since $a$ is also an extreme point of $P_{\rho}(G)^{1}$, there is an irreducible representation $\pi$ of the $C^{*}$-algebra $C_{\rho}^{*}(G)$ such that $a(x)=\langle\pi(x) \xi, \xi\rangle$ for some unit vector $\xi \in H_{\pi}$. Hence $\pi$ is an irreducible representation of $G$ as well. Thus, $a$ is also an extreme point of the unit ball of $P(G)[5]$ p.63]. Note that $a(e)=\|a\|=1$ since $a$ is a positive definite function. If $a=\frac{1}{2} b_{1}+\frac{1}{2} b_{2}$ for some $b_{1}$ and $b_{2}$ on the unit ball of $B(G)$, then $a(e)=1,\left|b_{1}(e)\right| \leq 1$ and $\left|b_{2}(e)\right| \leq 1$ imply that $b_{1}(e)=b_{2}(e)=1=\left\|b_{1}\right\|=\left\|b_{2}\right\|$. Hence both $b_{1}$ and $b_{2}$ are positive definite [11. So $b_{1}=b_{2}$. Therefore $a$ is an element of $\operatorname{Ext}\left(B(G)^{1}\right) \cap A(G)$.

In the proof of $(\mathrm{g}) \Rightarrow(\mathrm{b})$ above, we have actually proved the following theorem.

3.5. Theorem. Let $G$ be a locally compact group. If $B_{\rho}(G)$ has the $R N P$, then there are elements of $A(G)$ which are extreme points of the unit ball of $B(G)$.

Remarks. (1) If $B(G)$ has the RNP, then $B_{\rho}(G)$, being a subspace of $B(G)$, has the RNP, and so $\operatorname{Ext}\left(B(G)^{1}\right) \cap A(G) \neq \emptyset$ by Theorem 3.5. It is well known that there are non-compact groups $G$ for which $B(G)$ has the RNP (for example, Fell's group [14, Remark 4.6]). This shows that the condition $G$ be an INgroup in Theorem 3.4 above cannot be entirely dropped.

(2) In 2] Bekka et al. investigated whether the weak*-closedness of $A(G)$ in $B(G)$ implies the compactness of $G$. They proved that if $G$ contains an almost connected open normal subgroup, then $A(G)$ is weak*-closed in $B(G)$ if and only if $G$ is compact. The equivalence of (a) and (d) in Theorem 3.4 answers their question for IN-groups. Neither one of these results imply the other. In a private communication, Anthony Lau has informed us that he has a proof of the equivalence of (a) and (d) for IN-groups also, using the Kakutani-Kodira theorem and the RNP. However, our proof above does not use these results, and our Theorem 3.4 itself provides some other equivalent conditions.

(3) The equivalence of (a) and (f) is contained in Taylor's Theorem 4.7 in [14, and in view of the other equivalent conditions in our Theorem 3.4, our result is an improvement of Taylor's result. 


\section{ACKNOWLEDGMENT}

We thank the referee for kindly pointing out the following to us:

1. The proof of the implication $(\mathrm{g}) \Rightarrow(\mathrm{b})$ in Theorem 3.4 actually yields the following theorem.

Theorem 3.6. If $P_{\rho}(G)^{1}$ or $P(G)^{1}$ has a weak $k^{*}$-strongly exposed point, and $G$ is an IN-group, then $G$ must be compact.

2. Theorem 3.4 remains valid when (b) is replaced by

$\left(\mathrm{b}^{\prime}\right) \operatorname{Ext}\left(P(G)^{1}\right) \cap A(G) \neq \emptyset$.

3. The set $\operatorname{Ext}\left(P(G)^{1}\right)$ has been studied by A.C. Akemann and M.E. Walter, Non-abelian Pontryagin duality, Duke J. Math. 39 (1972), 451-463, as the dual of a non-abelian group $G$.

\section{REFERENCES}

1. G. Arsac, Sur l'espace de Banach engendré par les coefficients d'une représentation unitaire, Publ. Dép. Math. (Lyon) 13 (1976), 1-101. MR 56:3180

2. M.B. Bekka, E. Kaniuth, A.T. Lau and G. Schlichting, Weak*-closedness of subspaces of Fourier-Stieltjes algebras and weak ${ }^{*}$-continuity of the restriction map, Trans. Amer. Math. Soc. 350 (1998), 2277-2296. MR 98h:22004

3. A. Bélanger and B. Forrest, Geometric properties of coefficient function spaces determined by unitary representations of a locally compact groups, J. Math. Anal. Appl. 193 (1995), 390-405. MR 96f:22005

4. J. Diestel and J.J. UHL, Vector Measures, Mathematical Surveys, No. 15, Amer. Math. Soc., Rhode Island, 1977. MR 56:12216

5. J. Dixmier, $C^{*}$-Algebras, North-Holland, New York, 1977. MR 56:16388

6. P. Eymard, L'alg̀ebre de Fourier d'un groupe localement compact, Bull. Soc. Math. France 92 (1964), 181-236. MR 37:4208

7. C. Herz, Harmonic synthesis for subgroups, Ann. Inst. Fourier (Grenoble) 23 (1973), 91-123. MR 50:7956

8. A. T. Lau and A. Ülger, Some geometric properties on the Fourier and Fourier-Stieltjes algebras of locally compact groups, Arens regularity and related problems, Trans. Amer. Math. Soc. 337 (1993), 321-359. MR 93g:22007

9. T. Miao, Compactness of a locally compact group $G$ and geometric properties of $A_{p}(G)$, Can. J. Math. 48 (6) (1996), 1273-1285. MR 98g:43003

10. Decomposition of $B(G)$, to appear in Trans. Amer. Math. Soc.. CMP 98:09

11. G. J. Murphy, $C^{*}$-algebra and operator theory, Academic Press, San Diego, 1990. MR 91m:46084

12. T. W. Palmer, Classes of nonabelian, noncompact, locally compact groups, Rocky Mountain J. of Math. 8 (1973), 683-741. MR 81j:22003

13. K. Taylor, The type structure of the regular representation of a locally compact group, Math. Ann. 222 (6) (1976), 211-224. MR 54:12965

14. Geometry of the Fourier algebras and locally compact groups with atomic unitary representations, Math. Ann. 262 (1983), 183-190. MR 84h:43020

Department of Mathematics and Statistics, Lakehead University, Thunder Bay, Ontario, Canada P7B 5E1

E-mail address: pfmah@mist.lakeheadu.ca

E-mail address: tmiao@thunder.lakeheadu.ca 